\title{
Confidence Intervals for the Reciprocal of a Normal Mean with a Known Coefficient of Variation and a Restricted Parameter Space
}

\author{
Wararit Panichkitkosolkul \\ Department of Mathematics and Statistics \\ Thammasat University, Phathum Thani, Thailand \\ wararit@mathstat.sci.tu.ac.th
}

\begin{abstract}
The natural parameter space is known to be restricted in many real applications such as engineering, sciences and social sciences. The confidence interval derived from the classical Neyman procedure is unsatisfactory in the case of a restricted parameter space. New confidence intervals for the reciprocal of a normal mean with a known coefficient of variation and a restricted parameter space are proposed in this paper. A simulation study has been conducted to compare the performance of the proposed confidence intervals.
\end{abstract}

Keywords: Estimation, Normal distribution, Central tendency, Simulation.

Mathematics subject classification 62F25

\section{Introduction}

The reciprocal of a normal mean, defined by $\theta=\mu^{-1}$, where $\mu$ is the population mean, is widely used in many areas, such as experimental nuclear physics, biological sciences, agriculture and econometrics. For example, Lamanna et al. (1981) studied charged particle momentum, $p=\mu^{-1}$ where $\mu$ is the track curvature of a particle. The inverse of the common mean of structural econometric models was estimated by Zellner (1978). Furthermore, several researchers have studied the reciprocal of a normal mean. For example, Zaman (1981) proposed the estimators without moments in the case of the reciprocal of a normal mean. A class of estimators with a finite moment for the reciprocal of the mean was developed by Srivastava and Bhatnager (1981). Zaman (1985) also proposed the maximum likelihood estimate of the reciprocal of a normal mean with a class of zero-one loss functions. Voinov (1985) suggested the unbiased estimators of power for the reciprocal of the mean and related problems. Recently, Withers and Nadarajah (2013) discussed a theorem to construct the point estimators for the inverse powers of a normal mean. Two confidence intervals for the reciprocal of a normal mean with a known coefficient of variation were proposed by Wongkhao et al. (2013). Their confidence intervals can be applied when the coefficient of variation of a control group is known. The exact method was used to construct one of their confidence intervals from the pivotal statistics $Z$, where $Z$ follows the standard normal distribution. The other confidence interval is developed based on the generalized confidence interval (Weerahandi, 1993). However, the lower and upper limits of the confidence interval based on the exact method are difficult to compute since they depend on an infinite summation. Panichkitkosolkul (2017) proposed the approximate confidence interval for the reciprocal of a normal population mean with a known coefficient of variation. The approximate confidence interval performs as well as the exact confidence interval in 
terms of coverage probability. However, the approximate confidence interval is very easy to calculate compared with the exact confidence interval.

Although statistical inference is studied in a natural parameter space, the parameter space is restricted in several real applications, such as engineering, sciences and social sciences. For example, the blood pressure of patients or the weight of a human body are restricted or bounded. Furthermore, Mandelkern (2002) indicated the importance of statistical inference where the parameter space is known to be restricted. Additionally, he gave the example that the classical Neyman procedure is unsatisfactory in the case of a restricted parameter space. The main reason is that the information regarding the restriction is simply ignored. The other related works are Feldman and Cousins (1998) and Roe and Woodroofe (2001). Although research has been done on confidence intervals for the reciprocal of a normal mean, the confidence intervals for the reciprocal of a normal mean with a restricted parameter space have not been the subject of much study. Therefore, it would be of significant interest to develop confidence intervals for the reciprocal of a normal mean that include additional information on the population mean being restricted in order to improve the accuracy of the confidence interval. Motivated by the recent work of Panichkitkosolkul (2017), we propose confidence intervals for the reciprocal of a normal mean with a known coefficient of variation and a restricted parameter space in this paper.

The structure of this paper is as follows: Section 2 reviews two confidence intervals for the reciprocal of a normal mean with a known coefficient of variation. Confidence intervals for the reciprocal of a normal mean with a known coefficient of variation and a restricted parameter space are proposed in Section 3. The performance of all confidence intervals is investigated through a Monte Carlo simulation study in Section 4. We then conclude this paper in Section 5.

\section{Confidence Intervals for the Reciprocal of a Normal Mean with a Known Coefficient of Variation}

In this section, we review the confidence intervals for the reciprocal of a normal mean with a known coefficient of variation proposed recently by Wongkhao et al. (2013) and Panichkitkosolkul (2017). The exact and the approximate confidence intervals for the reciprocal of a normal mean with a known coefficient of variation are discussed.

The theorem and corollary proposed by Wongkhao et al. (2013) are reviewed and used to construct the exact confidence interval for $\theta$.

Theorem 1. (Wongkhao et al., 2013) Let $X_{1}, \ldots, X_{n}$ be a random sample of size $n$ from a normal distribution with mean $\mu$ and variance $\sigma^{2}$. The estimator of $\theta$ is $\hat{\theta}=\bar{X}^{-1}$ where $\bar{X}=n^{-1} \sum_{i=1}^{n} X_{i}$. The expectation of $\hat{\theta}$ and $\hat{\theta}^{2}$ when a coefficient of variation, $\tau=\sigma / \mu$ is known, are respectively

$$
E(\hat{\theta})=\theta\left[1+\sum_{k=1}^{\infty} \frac{(2 k) !}{2^{k} k !}\left(\frac{\tau^{2}}{n}\right)^{k}\right]
$$




$$
\alpha v \delta \quad E\left(\hat{\theta}^{2}\right)=\theta^{2} \sum_{k=1}^{\infty} \frac{(2 k+1) !}{2^{k} k !}\left(\frac{\tau^{2}}{n}\right)^{k}
$$

Proof of Theorem 1 See Wongkhao et al. (2013).

From (1), $\lim _{n \rightarrow \infty} E(\hat{\theta})=\theta$ and $E(\hat{\theta} / w)=\theta$, where $w=1+\sum_{k=1}^{\infty} \frac{(2 k) !}{2^{k} k !}\left(\frac{\tau^{2}}{n}\right)^{k}$. Therefore, the unbiased estimator of $\theta$ is $\hat{\theta} / w=(w \bar{X})^{-1}$.

Corollary 1. From Theorem $1, \operatorname{var}(\hat{\theta}) \approx \theta^{2} \tau^{2} / n$.

Proof of Corollary 1 See Wongkhao et al. (2013).

From the central limit theorem, we will use the fact that, $Z=\frac{\hat{\theta}-\theta}{\sqrt{\operatorname{var}(\hat{\theta})}} \square N(0,1)$. Based on Theorem 1 and Corollary 1 , we get

$$
Z=\frac{\hat{\theta} / w-\theta}{\sqrt{\theta^{2} \tau^{2} / n}} \square N(0,1)
$$

Therefore, the $100(1-\alpha) \%$ exact confidence interval for $\theta$ based on Equation (2) is

$$
C I_{\text {Exact }}=\left[(w \bar{X})^{-1}-z_{1-\alpha / 2} \sqrt{\tau^{2} / n \bar{X}^{2}}, \quad(w \bar{X})^{-1}+z_{1-\alpha / 2} \sqrt{\tau^{2} / n \bar{X}^{2}}\right],
$$

where $w=1+\sum_{k=1}^{\infty} \frac{(2 k) !}{2^{k} k !}\left(\frac{\tau^{2}}{n}\right)^{k}$ and $z_{1-\alpha / 2}$ is the $100(1-\alpha / 2)$ percentile of the standard normal distribution.

Next, the following theorem suggested by Panichkitkosolkul (2017) is shown to find the approximate confidence interval for $\theta$.

Theorem 2. Let $X_{1}, \ldots, X_{n}$ be a random sample of size $n$ from a normal distribution with mean $\mu$ and variance $\sigma^{2}$. The estimator of $\theta$ is $\hat{\theta}=\bar{X}^{-1}$ where $\bar{X}=n^{-1} \sum_{i=1}^{n} X_{i}$. The approximate expectation and variance of $\hat{\theta}$ when a coefficient of variation, $\tau=\sigma / \mu$ is known, are respectively

$$
E(\hat{\theta}) \approx \frac{1}{\mu}\left(1+\frac{\tau^{2}}{n}\right)
$$

and $\quad \operatorname{var}(\hat{\theta}) \approx \frac{\theta^{2} \tau^{2}}{n}$.

Proof of Theorem 2 See Panichkitkosolkul (2017).

It is clear from Equation (3) that $\hat{\theta}$ is asymptotically unbiased $\left(\lim _{n \rightarrow \infty} E(\hat{\theta})=\theta\right)$ and $E(\hat{\theta} / v)=\theta$, where $v=1+\tau^{2} / n$. Therefore, the unbiased estimator of $\theta$ is $\hat{\theta} / v=(v \bar{X})^{-1}$. 
From Equation (4), $\hat{\theta}$ is consistent $\left(\lim _{n \rightarrow \infty} \operatorname{var}(\hat{\theta})=0\right)$. Then, the central limit theorem and Theorem 2 are applied and we get,

$$
Z=\frac{\hat{\theta} / v-\theta}{\sqrt{\theta^{2} \tau^{2} / n}} \square N(0,1) .
$$

Therefore, it is easily seen that the $(1-\alpha) 100 \%$ approximate confidence interval for $\theta$ is

$$
C I_{\text {Approx }}=\left[(v \bar{X})^{-1}-z_{1-\alpha / 2} \sqrt{\tau^{2} / n \bar{X}^{2}}, \quad(v \bar{X})^{-1}+z_{1-\alpha / 2} \sqrt{\tau^{2} / n \bar{X}^{2}}\right]
$$

where $v=1+\tau^{2} / n$ and $z_{1-\alpha / 2}$ is the $100(1-\alpha / 2)$ percentile of the standard normal distribution.

\section{Confidence Intervals for the Reciprocal of a Normal Mean with a Known Coefficient of Variation and a Restricted Parameter Space}

Confidence intervals for the mean of a normal distribution with restricted parameter space were derived by Wang (2008). Following the method proposed by Wang (2008), we present confidence intervals for the reciprocal of a normal mean with a known coefficient of variation when the population mean is restricted.

The true value of a parameter of interest is usually unknown. However, parameter space is often known to be restricted and the bounds of parameter space are known. We denote $m_{1}$ and $m_{2}$ as the lower bound and the upper bound of the parameter space. When the parameter space is known to be restricted to the interval $\left(m_{1}, m_{2}\right)$, it is widely accepted that the confidence interval for a parameter $\beta$ is the confidence interval of the intersection between the interval $\left(m_{1}, m_{2}\right)$ and $\left[L_{\beta}, U_{\beta}\right]$, where $L_{\beta}$ and $U_{\beta}$ are the lower and upper limits of the confidence interval for $\beta$. Therefore, the confidence interval for $\beta$ when the parameter space is restricted, denoted as $C I_{B}$, is defined as

$$
C I_{B}=\left[\max \left(m_{1}, L_{\beta}\right), \min \left(m_{2}, U_{\beta}\right)\right] \text {. }
$$

Four possible confidence intervals in (5) are as follows:

1) if $m_{1}>L_{\beta}$ and $m_{2}>U_{\beta}$ then $C I_{B}$ is reduced to $C I_{B}=\left[m_{1}, U_{\beta}\right]$.

2) if $m_{1}>L_{\beta}$ and $m_{2}<U_{\beta}$ then $C I_{B}$ is reduced to $C I_{B}=\left[m_{1}, m_{2}\right]$.

3) if $m_{1}<L_{\beta}$ and $m_{2}>U_{\beta}$ then $C I_{B}$ is reduced to $C I_{B}=\left[L_{\beta}, U_{\beta}\right]$.

4) if $m_{1}<L_{\beta}$ and $m_{2}<U_{\beta}$ then $C I_{B}$ is reduced to $C I_{B}=\left[L_{\beta}, m_{2}\right]$.

When the parameter space of the population mean is $\left(m_{1}, m_{2}\right)$, straightforward calculation can show that the reciprocal of a normal mean is also restricted as follows:

$$
\begin{aligned}
& m_{1}<\mu<m_{2} \\
\Rightarrow & m_{2}^{-1}<\mu^{-1}<m_{1}^{-1}=\quad m_{2}^{-1}<\theta<m_{1}^{-1}
\end{aligned}
$$


According to Wang (2008) and Niwitpong (2013), the proposed confidence intervals for $\theta$ with a restricted mean are given by

$$
C I_{\theta}=\left[\max \left(m_{2}^{-1}, L_{\theta}\right), \min \left(m_{1}^{-1}, U_{\theta}\right)\right],
$$

where $L_{\theta}$ and $U_{\theta}$ are the lower and upper limits of the confidence intervals for $\theta$, respectively. In addition, the exact and approximate confidence intervals for $\theta$ reviewed in the previous section are used in order to obtain confidence intervals for $\theta$ when the population mean is restricted.

\section{Simulation Results}

The performances of the confidence intervals for the reciprocal of a normal mean with a known coefficient of variation and a restricted parameter space derived in the previous section were investigated through simulation studies in this section. A simulation was conducted using the R statistical software (Ihaka and Gentleman, 1996) version 3.3.2. The estimated coverage probabilities and expected lengths of two confidence intervals with unrestricted and restricted population means are demonstrated in Tables 1-6. The sets of normal data were generated with $\theta=0.1,0.2,0.5,1,5$ and 10 , and the coefficient of variation $\tau=0.05,0.10,0.20,0.33$ and 0.50 . The sample sizes were set at $n=10,20,30,50$ and 100. The parameter space of the population mean was set to the interval $(0.9 / \theta, 0.11 / \theta)$. The number of simulation runs was 10,000 and the nominal confidence level $1-\alpha$ was fixed at 0.95 .

In the simulation study, the estimated coverage probabilities of the confidence intervals with a restricted population mean are the same as those of the confidence intervals with an unrestricted population mean. Additionally, all confidence intervals have estimated coverage probabilities close to the nominal confidence level in most situations. The estimated coverage probabilities of all the confidence intervals do not increase or decrease according to the values of $\tau$ and $n$. The confidence intervals with a restricted population mean have shorter expected lengths than those of the confidence intervals with an unrestricted population mean when the sample sizes are not too large and the values of $\tau$ are not too small.

\section{Conclusion}

This paper proposes the confidence intervals for the reciprocal of a normal mean with a known coefficient of variation and a restricted parameter space. The new proposed confidence intervals are based on two existing confidence intervals: exact confidence interval and approximate confidence interval. Estimated coverage probabilities and the expected lengths of the confidence intervals are considered as the criteria of a good confidence interval. The simulation results point that the performances of proposed confidence intervals with a restricted population mean are the same as the performances of the confidence intervals with an unrestricted population mean in terms of coverage probability. However, the confidence intervals with a restricted population mean have the advantage of a shorter expected length in some situations. 


\section{Appendix: Source $\mathbf{R}$ code for all confidence intervals}

$$
\text { cal.w <-function(tao,n) }
$$

\{

temp $<-\operatorname{rep}(0,50)$

for $(\mathrm{k}$ in $1: 50)\{$

temp $[\mathrm{k}]<-\left(\text { factorial }\left(2^{*} \mathrm{k}\right) /\left(\left(2^{\wedge} \mathrm{k}\right)^{*} \text { factorial }(\mathrm{k})\right)\right)^{*}\left(\left(\left(\operatorname{tao}^{\wedge} 2\right) / \mathrm{n}\right)^{\wedge} \mathrm{k}\right)$

\}

w $<-1+\operatorname{sum}($ temp $)$

$\operatorname{return}(\mathrm{w})$

\}

ci.exact $<-$ function(y,tao,alpha)

\{

$\mathrm{n}<-$ length $(\mathrm{y})$

ybar $<-$ mean $(\mathrm{y})$

zeta.hat $<-1 /$ ybar

w $<-$ cal.w (tao,n)

$\mathrm{z}<-$ qnorm $(1-$ alpha/2)

$\mathrm{T} 1<-\left(\operatorname{tao}^{\wedge} 2\right) /\left(\mathrm{n}^{*}\left(\mathrm{ybar}^{\wedge} 2\right)\right)$

lower <- (zeta.hat/w)-z*sqrt(T1)

upper $<-\left(\right.$ zeta.hat/w) $+\mathrm{Z}^{*} \operatorname{sqrt}(\mathrm{T} 1)$

out $<$ - cbind(lower,upper)

return(out)

\}

ci.approx <-function(y,tao,alpha)

\{

$\mathrm{n}<-$ length $(\mathrm{y})$

ybar $<-\operatorname{mean}(\mathrm{y})$

zeta.hat $<-1 /$ ybar

$\mathrm{v}<-1+\left(\operatorname{tao}^{\wedge} 2\right) / \mathrm{n}$

$\mathrm{z}<-$ qnorm(1-alpha/2)

T1 <- $\left(\left(\text { zeta.hat }^{\wedge} 2\right)^{*}\left(\operatorname{tao}^{\wedge} 2\right)\right) / \mathrm{n}$

lower <- (zeta.hat/v)-z*sqrt(T1)

upper <- (zeta.hat/v)+z*sqrt(T1)

out $<$ - cbind(lower,upper)

return(out)

\}

ci.bounded <-function $(a, b, L, U)$

\{

lower <- $\max (1 / \mathrm{b}, \mathrm{L})$

upper $<-\min (1 / \mathrm{a}, \mathrm{U})$

out <- cbind(lower,upper)

return(out)

\} 


\section{Acknowledgement}

The author is grateful to the anonymous referees for their constructive comments and suggestions, which have significantly enhanced the quality and presentation of this paper.

\section{References}

1. Feldman, G.J. and Cousins, R.D. (1998). Unified approach to the classical statistical analysis of small signals. Physical Review D, 57, 3873-3889.

2. Ihaka, R. and Gentleman, R. (1996). R: A language for data analysis and graphics. Journal of Computational and Graphical Statistics, 5, 299-314.

3. Lamanna, E., Romano, G. and Sgrbi, C. (1981). Curvature measurements in nuclear emulsions. Nuclear Instruments and Methods, 187, 387-391.

4. Mandelkern, M. (2002). Setting confidence intervals for bounded parameters. Statistical Sciences, 17, 149-172.

5. Niwitpong, S. (2013). Confidence intervals for the mean of lognormal distribution with restricted parameter space. Applied Mathematical Sciences, 7, 161-166.

6. Panichkitkosolkul, W. (2017). Approximate confidence interval for the reciprocal of a normal mean with a known coefficient of variation. Metodološki zvezki (To appear).

7. Roe, B.P. and Woodroofe, M.B. (2001). Setting confidence belts, Physical Review $D, 60,3009-3015$.

8. Srivastava, V.K. and Bhatnager, S. (1981). Estimation of the inverse of mean. Journal of Statistical Planning and Inference, 5, 329-334.

9. Voinov, V.G. (1985). Unbiased estimation of powers of the inverse of mean and related problems. Sankhyā: The Indian Journal of Statistics, 47, 354-364.

10. Wang, H. (2008). Confidence intervals for the mean of a normal distribution with restricted parameter space, Journal of Statistical Computation and Simulation, 78, 829-841.

11. Weerahandi, S. (1993). Generalized confidence intervals. Journal of the American Statistical Association, 88, 899-905.

12. Withers, C.S. and Nadarajah, S. (2013). Estimators for the inverse powers of a normal mean. Journal of Statistical Planning and Inference, 143, 441-455.

13. Wongkhao, A., Niwitpong, S. and Niwitpong, S. (2013). Confidence interval for the inverse of a normal mean with a known coefficient of variation. International Journal of Mathematical, Computational, Statistical, Natural and Physical Engineer, 7, 877-880.

14. Zaman, A. (1981). Estimators without moments: the case of the reciprocal of a normal mean. Journal of Econometrics, 15, 289-298.

15. Zaman, A. (1985). Admissibility of the maximum likelihood estimate of the reciprocal of a normal mean with a class of zero-one loss functions. Sankhyā: The Indian Journal of Statistics, 47, 239-246.

16. Zellner, A. (1978). Estimation of functions of population means and regression coefficients including structural coefficients. Journal of Econometrics, 8, 127-158. 
Table 1: Estimated coverage probabilities and expected lengths of confidence intervals for the reciprocal of a normal mean with a known coefficient of variation and a restricted parameter space when $\theta=0.1$.

\begin{tabular}{|c|c|c|c|c|c|c|c|c|c|}
\hline \multirow{3}{*}{$n$} & \multirow{3}{*}{$\tau$} & \multicolumn{4}{|c|}{ Coverage Probabilities } & \multicolumn{4}{|c|}{ Expected Lengths } \\
\hline & & \multicolumn{2}{|c|}{$\begin{array}{l}\text { Unrestricted } \\
\text { Mean }\end{array}$} & \multicolumn{2}{|c|}{ Restricted Mean } & \multicolumn{2}{|c|}{$\begin{array}{l}\text { Unrestricted } \\
\text { Mean }\end{array}$} & \multicolumn{2}{|c|}{ Restricted Mean } \\
\hline & & Exact & Approx & Exact & Approx & Exact & Approx & Exact & Approx \\
\hline \multirow[t]{5}{*}{10} & 0.05 & 0.9514 & 0.9514 & 0.9514 & 0.9514 & 0.0062 & 0.0062 & 0.0062 & 0.0062 \\
\hline & 0.10 & 0.9488 & 0.9488 & 0.9488 & 0.9488 & 0.0124 & 0.0124 & 0.0120 & 0.0120 \\
\hline & 0.20 & 0.9514 & 0.9515 & 0.9514 & 0.9515 & 0.0249 & 0.0249 & 0.0170 & 0.0170 \\
\hline & 0.33 & 0.9490 & 0.9491 & 0.9490 & 0.9491 & 0.0418 & 0.0418 & 0.0183 & 0.0183 \\
\hline & 0.50 & 0.9361 & 0.9510 & 0.9361 & 0.9510 & 0.0637 & 0.0637 & 0.0183 & 0.0186 \\
\hline \multirow[t]{5}{*}{20} & 0.05 & 0.9512 & 0. & 0.9512 & 0 & 0. & 44 & 44 & 044 \\
\hline & 0.10 & 0.9503 & 0.9503 & 0.9503 & 0.9503 & 0.0088 & 0.0088 & 0.0088 & 0.0088 \\
\hline & 0.20 & 0.9484 & 0.9484 & 0.9484 & 0.9484 & 0.0176 & 0.0176 & 0.0150 & 0.0150 \\
\hline & 0.33 & 0.9486 & 0.9488 & 0.9486 & 0.9488 & 0.0294 & 0.0294 & 0.0175 & 0.0175 \\
\hline & 0.50 & 0.9478 & 0.9477 & 0.9478 & 0.9477 & 0.0444 & 0.0444 & 0.0184 & 0.0184 \\
\hline \multirow[t]{5}{*}{30} & 0.05 & 0.9464 & 0.9464 & 0.9464 & 0.9464 & 0.0036 & 0.0036 & 0.0036 & 0.0036 \\
\hline & 0.10 & 0.9521 & 0.9521 & 0.9521 & 0.9521 & 0.0072 & 0.0072 & 0.0072 & 0.0072 \\
\hline & 0.20 & 0.9498 & 0.9498 & 0.9498 & 0.9498 & 0.0143 & 0.0143 & 0.0134 & 0.0134 \\
\hline & 0.33 & 0.9467 & 0.9467 & 0.9467 & 0.9467 & 0.0240 & 0.0240 & 0.0169 & 0.0169 \\
\hline & 0.50 & 0.9464 & 0.9464 & 0.9464 & 0.9464 & 0.0361 & 0.0361 & 0.0180 & 0.0180 \\
\hline \multirow[t]{5}{*}{50} & 0.05 & 0.9524 & 0.9524 & 0.9524 & 0.9524 & 0.0028 & 0.0028 & 0.0028 & 0.0028 \\
\hline & 0.10 & 0.9490 & 0.9490 & 0.9490 & 0.9490 & 0.0055 & 0.0055 & 0.0055 & 0.0055 \\
\hline & 0.20 & 0.9482 & 0.9482 & 0.9482 & 0.9482 & 0.0111 & 0.0111 & 0.0109 & 0.0109 \\
\hline & 0.33 & 0.9517 & 0.9517 & 0.9517 & 0.9517 & 0.0185 & 0.0185 & 0.0154 & 0.0154 \\
\hline & 0.50 & 0.9521 & 0.9522 & 0.9521 & 0.9522 & 0.0278 & 0.0278 & 0.0175 & 0.0175 \\
\hline \multirow[t]{5}{*}{100} & 0.05 & 0.9507 & 0.9507 & 0.9507 & 0.9507 & 0.0020 & 0.0020 & 0.0020 & 0.0020 \\
\hline & 0.10 & 0.9492 & 0.9492 & 0.9492 & 0.9492 & 0.0039 & 0.0039 & 0.0039 & 0.0039 \\
\hline & 0.20 & 0.9489 & 0.9489 & 0.9489 & 0.9489 & 0.0078 & 0.0078 & 0.0078 & 0.0078 \\
\hline & 0.33 & 0.9486 & 0.9486 & 0.9486 & 0.9486 & 0.0131 & 0.0131 & 0.0125 & 0.0125 \\
\hline & 0.50 & 0.9498 & 0.9498 & 0.9498 & 0.9498 & 0.0196 & 0.0196 & 0.0158 & 0.0158 \\
\hline
\end{tabular}


Table 2: Estimated coverage probabilities and expected lengths of confidence intervals for the reciprocal of a normal mean with a known coefficient of variation and a restricted parameter space when $\theta=0.2$.

\begin{tabular}{|c|c|c|c|c|c|c|c|c|c|}
\hline \multirow{3}{*}{$n$} & \multirow{3}{*}{$\tau$} & \multicolumn{4}{|c|}{ Coverage Probabilities } & \multicolumn{4}{|c|}{ Expected Lengths } \\
\hline & & \multicolumn{2}{|c|}{$\begin{array}{l}\text { Unrestricted } \\
\text { Mean }\end{array}$} & \multicolumn{2}{|c|}{ Restricted Mean } & \multicolumn{2}{|c|}{$\begin{array}{c}\text { Unrestricted } \\
\text { Mean }\end{array}$} & \multicolumn{2}{|c|}{ Restricted Mean } \\
\hline & & Exact & Approx & Exact & Approx & Exact & Approx & Exact & Approx \\
\hline \multirow[t]{5}{*}{10} & 0.05 & 0.9527 & 0.9527 & 0.9527 & 0.9527 & 0.0124 & 0.0124 & 0.0124 & 0.0124 \\
\hline & 0.10 & 0.9535 & 0.9535 & 0.9535 & 0.9535 & 0.0248 & 0.0248 & 0.0241 & 0.0241 \\
\hline & 0.20 & 0.9524 & 0.9524 & 0.9524 & 0.9524 & 0.0498 & 0.0498 & 0.0341 & 0.0341 \\
\hline & 0.33 & 0.9457 & 0.9457 & 0.9457 & 0.9457 & 0.0836 & 0.0836 & 0.0365 & 0.0365 \\
\hline & 0.50 & 0.9335 & 0.9492 & 0.9335 & 0.9492 & 0.1273 & 0.1273 & 0.0365 & 0.0372 \\
\hline \multirow[t]{5}{*}{20} & 0.05 & 0.9492 & 0.9492 & 0.9492 & 0.9492 & 0.0088 & 0.0088 & 0.0088 & 0.0088 \\
\hline & 0.10 & 0.9495 & 0.9495 & 0.9495 & 0.9495 & 0.0175 & 0.0175 & 0.0175 & 0.0175 \\
\hline & 0.20 & 0.9497 & 0.9497 & 0.9497 & 0.9497 & 0.0352 & 0.0352 & 0.0302 & 0.0302 \\
\hline & 0.33 & 0.9535 & 0.9534 & 0.9535 & 0.9534 & 0.0587 & 0.0587 & 0.0351 & 0.0351 \\
\hline & 0.50 & 0.9497 & 0.9497 & 0.9497 & 0.9497 & 0.0888 & 0.0888 & 0.0368 & 0.0368 \\
\hline \multirow[t]{5}{*}{30} & 0.05 & 0.9513 & 0.9513 & 0.9513 & 0.9513 & 0.0072 & 0.0072 & 0.0072 & 0.0072 \\
\hline & 0.10 & 0.9500 & 0.9500 & 0.9500 & 0.9500 & 0.0143 & 0.0143 & 0.0143 & 0.0143 \\
\hline & 0.20 & 0.9499 & 0.9499 & 0.9499 & 0.9499 & 0.0287 & 0.0287 & 0.0268 & 0.0268 \\
\hline & 0.33 & 0.9517 & 0.9517 & 0.9517 & 0.9517 & 0.0479 & 0.0479 & 0.0336 & 0.0336 \\
\hline & 0.50 & 0.9509 & 0.9509 & 0.9509 & 0.9509 & 0.0722 & 0.0722 & 0.0361 & 0.0361 \\
\hline \multirow[t]{5}{*}{50} & 0.05 & 0.9474 & 0.9474 & 0.9474 & 0.9474 & 0.0055 & 0.0055 & 0.0055 & 0.0055 \\
\hline & 0.10 & 0.9509 & 0.9509 & 0.9509 & 0.9509 & 0.0111 & 0.0111 & 0.0111 & 0.0111 \\
\hline & 0.20 & 0.9493 & 0.9492 & 0.9493 & 0.9492 & 0.0222 & 0.0222 & 0.0219 & 0.0219 \\
\hline & 0.33 & 0.9487 & 0.9488 & 0.9487 & 0.9488 & 0.0370 & 0.0370 & 0.0308 & 0.0308 \\
\hline & 0.50 & 0.9499 & 0.9500 & 0.9499 & 0.9500 & 0.0557 & 0.0557 & 0.0349 & 0.0349 \\
\hline \multirow[t]{5}{*}{100} & 0.05 & 0.9491 & 0.9491 & 0.9491 & 0.9491 & 0.0039 & 0.0039 & 0.0039 & 0.0039 \\
\hline & 0.10 & 0.9478 & 0.9478 & 0.9478 & 0.9478 & 0.0078 & 0.0078 & 0.0078 & 0.0078 \\
\hline & 0.20 & 0.9507 & 0.9507 & 0.9507 & 0.9507 & 0.0157 & 0.0157 & 0.0157 & 0.0157 \\
\hline & 0.33 & 0.9490 & 0.9490 & 0.9490 & 0.9490 & 0.0262 & 0.0262 & 0.0251 & 0.0251 \\
\hline & 0.50 & 0.9491 & 0.9490 & 0.9491 & 0.9490 & 0.0393 & 0.0393 & 0.0316 & 0.0316 \\
\hline
\end{tabular}


Table 3: Estimated coverage probabilities and expected lengths of confidence intervals for the reciprocal of a normal mean with a known coefficient of variation and a restricted parameter space when $\theta=0.5$.

\begin{tabular}{|c|c|c|c|c|c|c|c|c|c|}
\hline \multirow{3}{*}{$n$} & \multirow{3}{*}{$\tau$} & \multicolumn{4}{|c|}{ Coverage Probabilities } & \multicolumn{4}{|c|}{ Expected Lengths } \\
\hline & & \multicolumn{2}{|c|}{$\begin{array}{l}\text { Unrestricted } \\
\text { Mean }\end{array}$} & \multicolumn{2}{|c|}{ Restricted Mean } & \multicolumn{2}{|c|}{$\begin{array}{l}\text { Unrestricted } \\
\text { Mean }\end{array}$} & \multicolumn{2}{|c|}{ Restricted Mean } \\
\hline & & Exact & Approx & Exact & Approx & Exact & Approx & Exact & Approx \\
\hline \multirow[t]{5}{*}{10} & 0.05 & 0.9529 & 0.9529 & 0.9529 & 0.9529 & 0.0310 & 0.0310 & 0.0310 & 0.0310 \\
\hline & 0.10 & 0.9498 & 0.9498 & 0.9498 & 0.9498 & 0.0621 & 0.0621 & 0.0602 & 0.0602 \\
\hline & 0.20 & 0.9462 & 0.9463 & 0.9462 & 0.9463 & 0.1245 & 0.1245 & 0.0850 & 0.0850 \\
\hline & 0.33 & 0.9499 & 0.9501 & 0.9499 & 0.9501 & 0.2090 & 0.2090 & 0.0914 & 0.0914 \\
\hline & 0.50 & 0.9338 & 0.9468 & 0.9338 & 0.9468 & 0.3180 & 0.3180 & 0.0913 & 0.0930 \\
\hline \multirow[t]{5}{*}{20} & 0.05 & 0.9501 & 0.9501 & 0.9501 & 0.9501 & 0.0219 & 0.0219 & 0.0219 & 0.0219 \\
\hline & 0.10 & 0.9512 & 0.9512 & 0.9512 & 0.9512 & 0.0438 & 0.0438 & 0.0438 & 0.0438 \\
\hline & 0.20 & 0.9486 & 0.9486 & 0.9486 & 0.9486 & 0.0878 & 0.0878 & 0.0754 & 0.0754 \\
\hline & 0.33 & 0.9465 & 0.9465 & 0.9465 & 0.9465 & 0.1470 & 0.1470 & 0.0876 & 0.0876 \\
\hline & 0.50 & 0.9486 & 0.9485 & 0.9486 & 0.9485 & 0.2223 & 0.2223 & 0.0920 & 0.0920 \\
\hline \multirow[t]{5}{*}{30} & 0.05 & 0.9514 & 0.9514 & 0.9514 & 0.9514 & 0.0179 & 0.0179 & 0.0179 & 0.0179 \\
\hline & 0.10 & 0.9456 & 0.9456 & 0.9456 & 0.9456 & 0.0358 & 0.0358 & 0.0358 & 0.0358 \\
\hline & 0.20 & 0.9502 & 0.9502 & 0.9502 & 0.9502 & 0.0717 & 0.0717 & 0.0669 & 0.0669 \\
\hline & 0.33 & 0.9464 & 0.9464 & 0.9464 & 0.9464 & 0.1197 & 0.1197 & 0.0841 & 0.0841 \\
\hline & 0.50 & 0.9528 & 0.9529 & 0.9528 & 0.9529 & 0.1805 & 0.1805 & 0.0905 & 0.0905 \\
\hline \multirow[t]{5}{*}{50} & 0.05 & 0.9506 & 0.9506 & 0.9506 & 0.9506 & 0.0139 & 0.0139 & 0.0139 & 0.0139 \\
\hline & 0.10 & 0.9520 & 0.9520 & 0.9520 & 0.9520 & 0.0277 & 0.0277 & 0.0277 & 0.0277 \\
\hline & 0.20 & 0.9483 & 0.9483 & 0.9483 & 0.9483 & 0.0555 & 0.0555 & 0.0547 & 0.0547 \\
\hline & 0.33 & 0.9512 & 0.9513 & 0.9512 & 0.9513 & 0.0926 & 0.0926 & 0.0773 & 0.0773 \\
\hline & 0.50 & 0.9484 & 0.9484 & 0.9484 & 0.9484 & 0.1392 & 0.1392 & 0.0869 & 0.0869 \\
\hline \multirow[t]{5}{*}{100} & 0.05 & 0.9478 & 0.9478 & 0.9478 & 0.9478 & 0.0098 & 0.0098 & 0.0098 & 0.0098 \\
\hline & 0.10 & 0.9493 & 0.9493 & 0.9493 & 0.9493 & 0.0196 & 0.0196 & 0.0196 & 0.0196 \\
\hline & 0.20 & 0.9507 & 0.9507 & 0.9507 & 0.9507 & 0.0392 & 0.0392 & 0.0392 & 0.0392 \\
\hline & 0.33 & 0.9522 & 0.9522 & 0.9522 & 0.9522 & 0.0654 & 0.0654 & 0.0628 & 0.0628 \\
\hline & 0.50 & 0.9533 & 0.9532 & 0.9533 & 0.9532 & 0.0982 & 0.0982 & 0.0789 & 0.0789 \\
\hline
\end{tabular}


Table 4: Estimated coverage probabilities and expected lengths of confidence intervals for the reciprocal of a normal mean with a known coefficient of variation and a restricted parameter space when $\theta=1$.

\begin{tabular}{|c|c|c|c|c|c|c|c|c|c|}
\hline \multirow{3}{*}{$n$} & \multirow{3}{*}{$\tau$} & \multicolumn{4}{|c|}{ Coverage Probabilities } & \multicolumn{4}{|c|}{ Expected Lengths } \\
\hline & & \multicolumn{2}{|c|}{$\begin{array}{l}\text { Unrestricted } \\
\text { Mean }\end{array}$} & \multicolumn{2}{|c|}{ Restricted Mean } & \multicolumn{2}{|c|}{$\begin{array}{l}\text { Unrestricted } \\
\text { Mean }\end{array}$} & \multicolumn{2}{|c|}{ Restricted Mean } \\
\hline & & Exact & Approx & Exact & Approx & Exact & Approx & Exact & Approx \\
\hline \multirow[t]{5}{*}{10} & 0.05 & 0.9500 & 0.9500 & 0.9500 & 0.9500 & 0.0620 & 0.0620 & 0.0620 & 0.0620 \\
\hline & 0.10 & 0.9496 & 0.9496 & 0.9496 & 0.9496 & 0.1241 & 0.1241 & 0.1205 & 0.1205 \\
\hline & 0.20 & 0.9507 & 0.9509 & 0.9507 & 0.9509 & 0.2489 & 0.2489 & 0.1704 & 0.1704 \\
\hline & 0.33 & 0.9483 & 0.9484 & 0.9483 & 0.9484 & 0.4175 & 0.4175 & 0.1828 & 0.1829 \\
\hline & 0.50 & 0.9302 & 0.9450 & 0.9302 & 0.9450 & 0.6368 & 0.6368 & 0.1824 & 0.1859 \\
\hline \multirow[t]{5}{*}{20} & 0.05 & 0.9476 & 0.9476 & 0.9476 & 0.9476 & 0.0438 & 0.0438 & 0.0438 & 0.0438 \\
\hline & 0.10 & 0.9510 & 0.9509 & 0.9510 & 0.9509 & 0.0877 & 0.0877 & 0.0876 & 0.0876 \\
\hline & 0.20 & 0.9505 & 0.9505 & 0.9505 & 0.9505 & 0.1758 & 0.1758 & 0.1508 & 0.1508 \\
\hline & 0.33 & 0.9536 & 0.9539 & 0.9536 & 0.9539 & 0.2935 & 0.2935 & 0.1757 & 0.1757 \\
\hline & 0.50 & 0.9440 & 0.9440 & 0.9440 & 0.9440 & 0.4441 & 0.4441 & 0.1830 & 0.1830 \\
\hline \multirow[t]{5}{*}{30} & 0.05 & 0.9494 & 0.9494 & 0.9494 & 0.9494 & 0.0358 & 0.0358 & 0.0358 & 0.0358 \\
\hline & 0.10 & 0.9487 & 0.9487 & 0.9487 & 0.9487 & 0.0716 & 0.0716 & 0.0716 & 0.0716 \\
\hline & 0.20 & 0.9533 & 0.9533 & 0.9533 & 0.9533 & 0.1433 & 0.1433 & 0.1338 & 0.1338 \\
\hline & 0.33 & 0.9508 & 0.9509 & 0.9508 & 0.9509 & 0.2395 & 0.2395 & 0.1683 & 0.1683 \\
\hline & 0.50 & 0.9477 & 0.9477 & 0.9477 & 0.9477 & 0.3600 & 0.3600 & 0.1796 & 0.1797 \\
\hline \multirow[t]{5}{*}{50} & 0.05 & 0.9467 & 0.9467 & 0.9467 & 0.9467 & 0.0277 & 0.0277 & 0.0277 & 0.0277 \\
\hline & 0.10 & 0.9491 & 0.9491 & 0.9491 & 0.9491 & 0.0555 & 0.0555 & 0.0555 & 0.0555 \\
\hline & 0.20 & 0.9457 & 0.9457 & 0.9457 & 0.9457 & 0.1109 & 0.1109 & 0.1094 & 0.1094 \\
\hline & 0.33 & 0.9502 & 0.9502 & 0.9502 & 0.9502 & 0.1853 & 0.1853 & 0.1543 & 0.1543 \\
\hline & 0.50 & 0.9521 & 0.9520 & 0.9521 & 0.9520 & 0.2785 & 0.2785 & 0.1745 & 0.1745 \\
\hline \multirow[t]{5}{*}{100} & 0.05 & 0.9487 & 0.9487 & 0.9487 & 0.9487 & 0.0196 & 0.0196 & 0.0196 & 0.0196 \\
\hline & 0.10 & 0.9493 & 0.9493 & 0.9493 & 0.9493 & 0.0392 & 0.0392 & 0.0392 & 0.0392 \\
\hline & 0.20 & 0.9506 & 0.9506 & 0.9506 & 0.9506 & 0.0784 & 0.0784 & 0.0784 & 0.0784 \\
\hline & 0.33 & 0.9498 & 0.9498 & 0.9498 & 0.9498 & 0.1308 & 0.1308 & 0.1254 & 0.1254 \\
\hline & 0.50 & 0.9486 & 0.9486 & 0.9486 & 0.9486 & 0.1966 & 0.1966 & 0.1584 & 0.1584 \\
\hline
\end{tabular}


Table 5: Estimated coverage probabilities and expected lengths of confidence intervals for the reciprocal of a normal mean with a known coefficient of variation and a restricted parameter space when $\theta=5$.

\begin{tabular}{|c|c|c|c|c|c|c|c|c|c|}
\hline \multirow{3}{*}{$n$} & \multirow{3}{*}{$\tau$} & \multicolumn{4}{|c|}{ Coverage Probabilities } & \multicolumn{4}{|c|}{ Expected Lengths } \\
\hline & & \multicolumn{2}{|c|}{$\begin{array}{l}\text { Unrestricted } \\
\text { Mean }\end{array}$} & \multicolumn{2}{|c|}{ Restricted Mean } & \multicolumn{2}{|c|}{$\begin{array}{l}\text { Unrestricted } \\
\text { Mean }\end{array}$} & \multicolumn{2}{|c|}{ Restricted Mean } \\
\hline & & Exact & Approx & Exact & Approx & Exact & Approx & Exact & Approx \\
\hline \multirow[t]{5}{*}{10} & 0.05 & 0.9495 & 0.9494 & 0.9495 & 0.9494 & 0.3100 & 0.3100 & 0.3100 & 0.3100 \\
\hline & 0.10 & 0.9518 & 0.9518 & 0.9518 & 0.9518 & 0.6205 & 0.6205 & 0.6026 & 0.6026 \\
\hline & 0.20 & 0.9495 & 0.9495 & 0.9495 & 0.9495 & 1.2445 & 1.2445 & 0.8515 & 0.8515 \\
\hline & 0.33 & 0.9445 & 0.9447 & 0.9445 & 0.9447 & 2.0937 & 2.0937 & 0.9145 & 0.9147 \\
\hline & 0.50 & 0.9337 & 0.9434 & 0.9337 & 0.9434 & 3.1929 & 3.1929 & 0.9145 & 0.9308 \\
\hline \multirow[t]{5}{*}{20} & 0.05 & 0.9499 & 0.9499 & 0.9499 & 0. & 0. & 1 & 0.2191 & 1 \\
\hline & 0.10 & 0.9454 & 0.9454 & 0.9454 & 0.9 & 0. & 0.4384 & 0.4378 & 1378 \\
\hline & 0.20 & 0.9501 & 0.9502 & 0.9501 & 0.9502 & 0.8786 & 0.8786 & 0.7540 & 0.7540 \\
\hline & 0.33 & 0.9461 & 0.9462 & 0.9461 & 0.9462 & 1.4700 & 1.4700 & 0.8769 & 0.8770 \\
\hline & 0.50 & 0.9492 & 0.9494 & 0.9492 & 0.9494 & 2.2171 & 2.2171 & 0.9176 & 0.9179 \\
\hline \multirow[t]{5}{*}{30} & 0.05 & 0.9489 & 0.9489 & 0.9489 & 0.9489 & 0.1789 & 0.1789 & 0.1789 & 0.1789 \\
\hline & 0.10 & 0.9456 & 0.9456 & 0.9456 & 0.9456 & 0.3579 & 0.3579 & 0.3579 & 0.3579 \\
\hline & 0.20 & 0.9503 & 0.9503 & 0.9503 & 0.9503 & 0.7171 & 0.7171 & 0.6704 & 0.6704 \\
\hline & 0.33 & 0.9478 & 0.9478 & 0.9478 & 0.9478 & 1.1976 & 1.1976 & 0.8408 & 0.8409 \\
\hline & 0.50 & 0.9487 & 0.9487 & 0.9487 & 0.9487 & 1.8049 & 1.8049 & 0.9037 & 0.9038 \\
\hline \multirow[t]{5}{*}{50} & 0.05 & 0.9495 & 0.9495 & 0.9495 & 0.9495 & 0.1386 & 0.1386 & 0.1386 & 0.1386 \\
\hline & 0.10 & 0.9508 & 0.9508 & 0.9508 & 0.9508 & 0.2772 & 0.2772 & 0.2772 & 0.2772 \\
\hline & 0.20 & 0.9497 & 0.9497 & 0.9497 & 0.9497 & 0.5548 & 0.5548 & 0.5474 & 0.5474 \\
\hline & 0.33 & 0.9477 & 0.9477 & 0.9477 & 0.9477 & 0.9258 & 0.9258 & 0.7695 & 0.7695 \\
\hline & 0.50 & 0.9489 & 0.9488 & 0.9489 & 0.9488 & 1.3940 & 1.3940 & 0.8723 & 0.8723 \\
\hline \multirow[t]{5}{*}{100} & 0.05 & 0.9501 & 0.9501 & 0.9501 & 0.9501 & 0.0980 & 0.0980 & 0.0980 & 0.0980 \\
\hline & 0.10 & 0.9477 & 0.9477 & 0.9477 & 0.9477 & 0.1960 & 0.1960 & 0.1960 & 0.1960 \\
\hline & 0.20 & 0.9534 & 0.9534 & 0.9534 & 0.9534 & 0.3921 & 0.3921 & 0.3920 & 0.3920 \\
\hline & 0.33 & 0.9510 & 0.9510 & 0.9510 & 0.9510 & 0.6542 & 0.6542 & 0.6282 & 0.6282 \\
\hline & 0.50 & 0.9480 & 0.9480 & 0.9480 & 0.9480 & 0.9834 & 0.9834 & 0.7889 & 0.7889 \\
\hline
\end{tabular}


Table 6: Estimated coverage probabilities and expected lengths of confidence intervals for the reciprocal of a normal mean with a known coefficient of variation and a restricted parameter space when $\theta=10$.

\begin{tabular}{|c|c|c|c|c|c|c|c|c|c|}
\hline \multirow{3}{*}{$n$} & \multirow{3}{*}{$\tau$} & \multicolumn{4}{|c|}{ Coverage Probabilities } & \multicolumn{4}{|c|}{ Expected Lengths } \\
\hline & & \multicolumn{2}{|c|}{$\begin{array}{l}\text { Unrestricted } \\
\text { Mean }\end{array}$} & \multicolumn{2}{|c|}{ Restricted Mean } & \multicolumn{2}{|c|}{$\begin{array}{l}\text { Unrestricted } \\
\text { Mean }\end{array}$} & \multicolumn{2}{|c|}{ Restricted Mean } \\
\hline & & Exact & Approx & Exact & Approx & Exact & Approx & Exact & Approx \\
\hline \multirow[t]{5}{*}{10} & 0.05 & 0.9536 & 0.9536 & 0.9536 & 0.9536 & 0.6199 & 0.6199 & 0.6199 & 0.6199 \\
\hline & 0.10 & 0.9511 & 0.9511 & 0.9511 & 0.9511 & 1.2407 & 1.2407 & 1.2048 & 1.2048 \\
\hline & 0.20 & 0.9491 & 0.9491 & 0.9491 & 0.9491 & 2.4886 & 2.4886 & 1.6963 & 1.6964 \\
\hline & 0.33 & 0.9507 & 0.9507 & 0.9507 & 0.9507 & 4.1854 & 4.1854 & 1.8309 & 1.8312 \\
\hline & 0.50 & 0.9324 & 0.9470 & 0.9324 & 0.9470 & 6.3649 & 6.3649 & 1.8261 & 1.8630 \\
\hline \multirow[t]{5}{*}{20} & 0.05 & 9517 & 0.9517 & 0.95 & 0.95 & 0.4383 & 0. & 3 & 0.4383 \\
\hline & 0.10 & 0.9491 & 0.9491 & 0.9491 & 0.94 & 3771 & 71 & 61 & 0.8761 \\
\hline & 0.20 & 0.9486 & 0.9486 & 0.9486 & 0.9486 & 7574 & 1.7574 & 1.5028 & 1.5029 \\
\hline & 0.33 & 0.9479 & 0.9479 & 0.9479 & 0.9479 & 2.9372 & 2.9372 & 1.7534 & 1.7535 \\
\hline & 0.50 & 0.9468 & 0.9473 & 0.9468 & 0.9473 & 4.4429 & 4.4429 & 1.8295 & 1.8299 \\
\hline \multirow[t]{5}{*}{30} & 0.05 & 0.9516 & 0.9516 & 0.95 & 0.95 & 0.3578 & 0.3578 & 0.35 & 0.3578 \\
\hline & 0.10 & 0.9482 & 0.9482 & 0.9482 & 0.9482 & 0.7159 & 0.7159 & 0.7158 & 0.7158 \\
\hline & 0.20 & 0.9533 & 0.9534 & 0.9533 & 0.9534 & 1.4328 & 1.4328 & 1.3411 & 1.3411 \\
\hline & 0.33 & 0.9475 & 0.9477 & 0.9475 & 0.9477 & 2.3963 & 2.3963 & 1.6845 & 1.6846 \\
\hline & 0.50 & 0.9489 & 0.9489 & 0.9489 & 0.9489 & 3.6109 & 3.6109 & 1.8072 & 1.8074 \\
\hline \multirow[t]{5}{*}{50} & 0.05 & 0.9532 & 0.9532 & 0.9532 & 0.9532 & 0.2772 & 0.2772 & 0.2772 & 0.2772 \\
\hline & 0.10 & 0.9522 & 0.9522 & 0.9522 & 0.9522 & 0.5544 & 0.5544 & 0.5544 & 0.5544 \\
\hline & 0.20 & 0.9496 & 0.9496 & 0.9496 & 0.9496 & 1.1097 & 1.1097 & 1.0950 & 1.0950 \\
\hline & 0.33 & 0.9494 & 0.9493 & 0.9494 & 0.9493 & 1.8524 & 1.8524 & 1.5404 & 1.5404 \\
\hline & 0.50 & 0.9501 & 0.9501 & 0.9501 & 0.9501 & 2.7872 & 2.7872 & 1.7393 & 1.7394 \\
\hline \multirow[t]{5}{*}{100} & 0.05 & 0.9523 & 0.9523 & 0.9523 & 0.9523 & 0.1960 & 0.1960 & 0.1960 & 0.1960 \\
\hline & 0.10 & 0.9487 & 0.9487 & 0.9487 & 0.9487 & 0.3920 & 0.3920 & 0.3920 & 0.3920 \\
\hline & 0.20 & 0.9508 & 0.9508 & 0.9508 & 0.9508 & 0.7840 & 0.7840 & 0.7838 & 0.7838 \\
\hline & 0.33 & 0.9477 & 0.9477 & 0.9477 & 0.9477 & 1.3084 & 1.3084 & 1.2545 & 1.2545 \\
\hline & 0.50 & 0.9490 & 0.9490 & 0.9490 & 0.9490 & 1.9659 & 1.9659 & 1.5791 & 1.5791 \\
\hline
\end{tabular}

